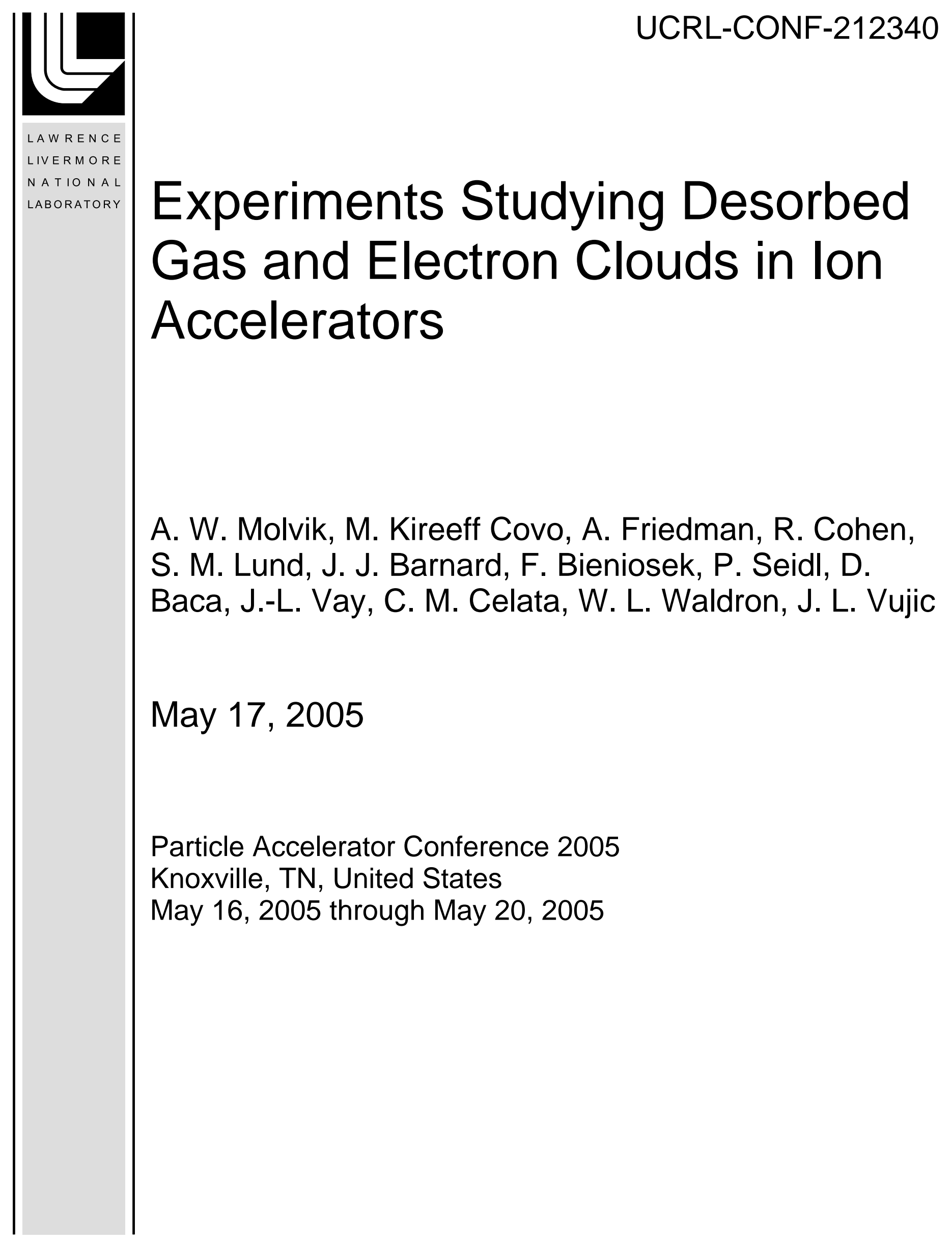


This document was prepared as an account of work sponsored by an agency of the United States Government. Neither the United States Government nor the University of California nor any of their employees, makes any warranty, express or implied, or assumes any legal liability or responsibility for the accuracy, completeness, or usefulness of any information, apparatus, product, or process disclosed, or represents that its use would not infringe privately owned rights. Reference herein to any specific commercial product, process, or service by trade name, trademark, manufacturer, or otherwise, does not necessarily constitute or imply its endorsement, recommendation, or favoring by the United States Government or the University of California. The views and opinions of authors expressed herein do not necessarily state or reflect those of the United States Government or the University of California, and shall not be used for advertising or product endorsement purposes. 


\title{
EXPERIMENTS STUDYING DESORBED GAS AND ELECTRON CLOUDS IN ION ACCELERATORS*
}

\author{
A.W.Molvik**, M. Kireeff Covo, A. Friedman, R. Cohen, S.M. Lund, J.J. Barnard, LLNL, \\ Livermore, CA 94550, U.S.A.
F. Bieniosek, P. Seidl, D. Baca, J.-L. Vay, C.M. Celata, W.L. Waldron, LBNL, Berkeley, CA
94720-8201, U.S.A.
J.L. Vujic, Univ. California, Berkeley, CA 94720, U.S.A.

\begin{abstract}
Electron clouds and gas pressure rise limit the performance of many major accelerator rings. We are studying these issues experimentally with $\sim 1 \mathrm{MeV}$ heavyion beams, coordinated with significant efforts in selfconsistent simulation and theory. The experiments use multiple diagnostics, within and between quadrupole magnets, to measure the sources and accumulation of electrons and gas. In support of these studies, we have measured gas desorption and electron emission coefficients for potassium ions impinging on stainless steel targets at angles near grazing incidence. Our goal is to measure the electron particle balance for each source ionization of gas, emission from beam tubes, and emission from an end wall - determine the electron effects on the ion beam and apply the increased understanding to mitigation. We describe progress towards that goal.
\end{abstract}

\section{INTRODUCTION}

Electron clouds and gas pressure rise limit the performance of many major accelerator rings, and may limit linacs being developed as drivers for heavy-ioninertial fusion (HIF) and for high-energy-density physics (HEDP). For the last three years, we have had a multilaboratory effort to understand the underlying physics through the coordinated application of experiment, theory, and simulation. This paper emphasizes the experimental component, accompanying papers discuss the theory [1,2] and simulations [3].

Electron cloud effects generally occur gradually, over many passes of a beam through an accelerator ring. However, we have demonstrated in both experiment and simulation that, electron densities approaching the beam density can significantly degrade beam properties in the short distance of 2 lattice periods in linac. We study these effects in the High-Current Experiment (HCX), shown in Fig. 1. A suppressor ring electrode, surrounding the beam after it exits the last quadrupole magnet, can be biased to $-10 \mathrm{kV}$ to prevent ion-induced electron emission off an end wall (a slit plate) from reaching the magnets, or can be left unbiased to allow electrons to be emitted from the

\footnotetext{
*This work was performed under the auspices of the U.S. Department of Energy by University of California, LLNL and LBNL under contracts W-7405-Eng-48, and DE-AC03-76F00098.

**molvik1@1lnl.gov
}

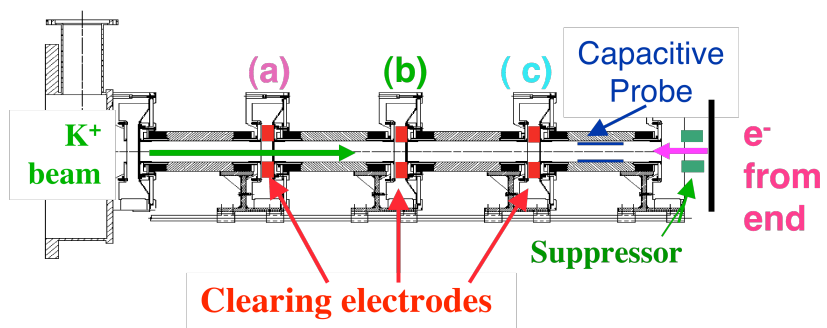

Figure 1. HCX in region of 4 quadrupole magnets, with clearing electrode rings between magnets and a suppressor electrode ring after the last magnet.
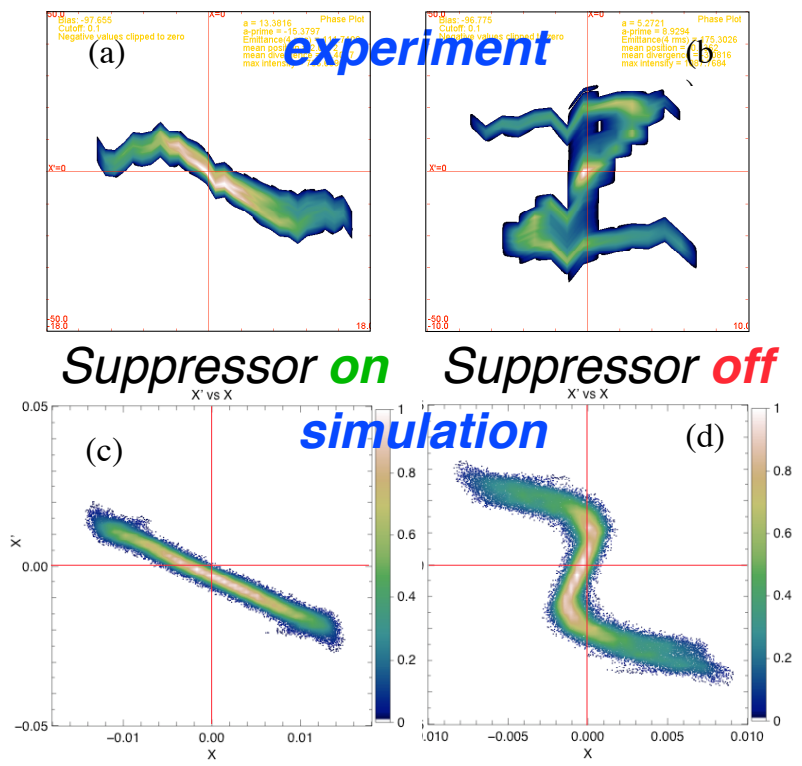

Figure 2. Optical slit scanner plots of ion beam phase space $X, X^{\prime}$. (a) Experiment with electron suppressor on. (b) Experiment with electron suppressor off. (c) Simulation for electron suppressor on for no electrons. (d) Simulation with electron suppressor off.

end wall and to flow into the magnets. An example of the effects of the suppressor on and off is shown in Fig. 2.

With the suppressor on, the experiment shows kinks at the ends of the XX' phase space plots with a slight " $Z$ " 
behavior, perhaps because electrons from other sources are present. (In the last section, we will return to the question of how many electrons are present.) The raw data show the beam degradation clearly, with the slit in the center of the "Z", we see a triple image of the slit. Simulations, with the suppressor on, resulting in no electrons in the beam, show a well-behaved ion beam with no Z-like kinks in the phase-space distribution. Simulations with the suppressor off allow electron densities to approach the beam density, which produces significant effects on the beam, quantitatively reproducing the dominant Z-phase space. Further details are available in Refs. [1,3].

\section{GAS DESORPTION \& ELECTRON EMISSION}

Heavy-ion accelerators are particularly prone to gas desorption caused by beam loss to walls. Surprisingly large desorption coefficients of 10,000 have been measured for $1 \mathrm{MeV} \mathrm{K}^{+}$ions in the high vacuum $\mathrm{HCX}$ [4], and for $800 \mathrm{MeV}$ lead ions in the ultra-high vacuum CERN LINAC 3 [5]. Desorbed gas can generate significant electron clouds, particularly in the injection through low-energy transport range, where ionization cross sections are large. Indeed, in heavy ion fusion
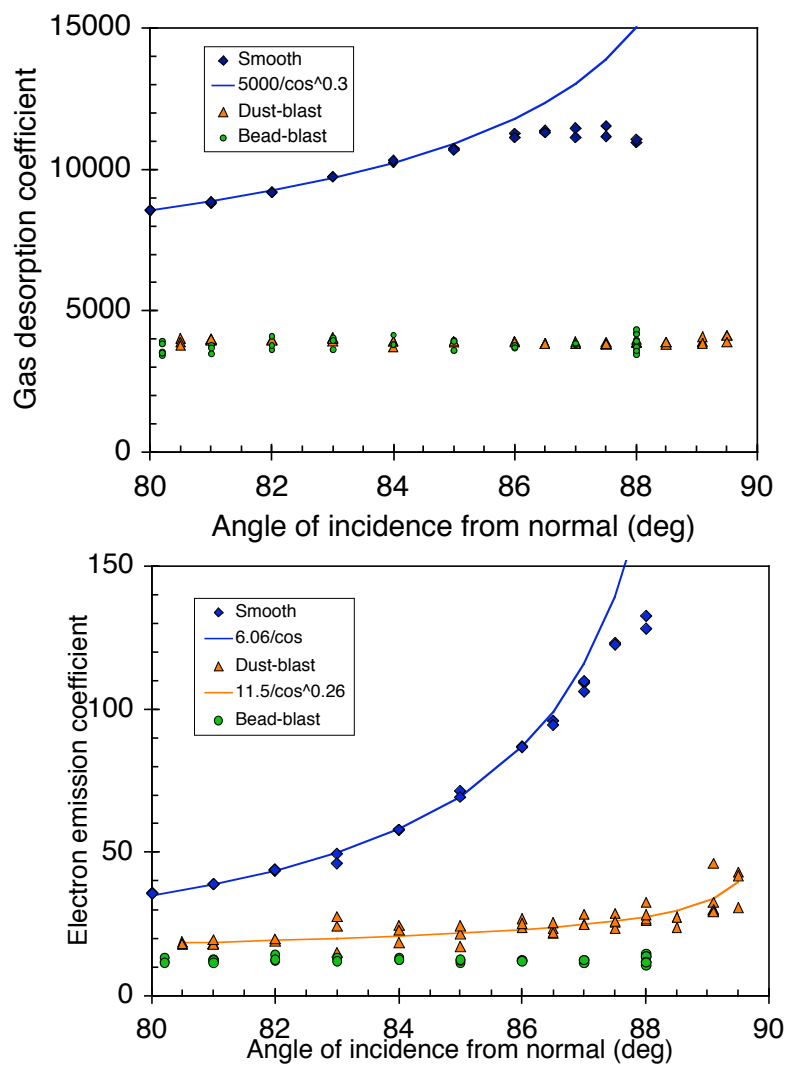

Fig. 3. (a) Gas desorption coefficients vs. ion angle of incidence for 3 surfaces. (b) Same for electron emission coefficients. accelerators, we expect beam-ionization of desorbed gas to be the dominant source of electrons.

Significant electron emission is also produced by heavy ions, striking a beam tube near grazing incidence. This scales as $1 / \cos (\theta)$ with the ion angle of incidence $\theta$, measured from normal to the surface, as shown in references $[4,6]$. We argued that roughened surfaces could reduce electron emission; because, instead of striking a smooth surface near grazing incidence, a grazingincidence ion would strike the rims of craters on the rough surface at angles far from grazing.

In Ref. [4], we showed that a surface, roughened by glass-bead-blasting, reduced gas desorption by a factor of 2 and electron emission by a factor of 10. This is effective at low ion energies where the range of the ion is less than the crater rim thickness. At high energies, where a grazing incidence ion might penetrate multiple crater rims, this would be more likely to increase gas desorption.

Bead blasting worked well on thick surfaces $(\geq 1.5 \mathrm{~mm}$ thick), but caused severe warping of thin $(\leq 0.5 \mathrm{~mm}$ thick) stainless steel. As a gentler substitute, we used a dental aluminum-oxide dust blaster. Under magnification, the surface appeared as rough as with bead blasting, however, thin sheet metal did not warp significantly when the blasting was done quickly and carefully. The results are shown in Fig. 3, where we compare bead-blasted and dust-blasted surfaces. The dust-blasting is seen to gain most of the mitigation advantages of the bead-blasted surface in reducing gas desorption as well as did bead blasting; and in reducing electron emission by a factor of four.

\section{ELECTRON SOURCE/ACCUMULATION DIAGNOSTICS}

Electrons can be generated from three sources: ionization of gas, emission from a beam tube, and in a linac - emission from an end wall. We have developed diagnostics to measure each of these.

The HCX in the region of four magnetic quadrupoles is shown in Fig. 1. To the left is the D2 diagnostic region



Fig. 4. Elliptical cross-section magnet tube with octagonal diagnostics tube installed. Full-length electrodes are end of each magnetic field line. 
between 10 electrostatic quadrupoles and the 4 magnetic quadrupoles. Each magnetic quadrupole has $30 \mathrm{~cm}$ long magnetic field coils in a $47 \mathrm{~cm}$ length elliptical tube that has major and minor inner radii of $5 \mathrm{~cm}$ and $3 \mathrm{~cm}$ respectively. Between each pair of magnets, and after the last one, diagnostic access is provided in a $5 \mathrm{~cm}$ gap, each with 7 ports. Arrays of diagnostics are mounted on the outside of octagonal tubes that fit within the elliptical quadrupole-magnet beam tubes, Fig. 4. Two different arrays are placed within the third and fourth quadrupoles. A gap of about $0.7 \mathrm{~cm}$ annular space is provided between the octagonal diagnostics mounting tube and the elliptical magnet bore for the recessed diagnostics and cables. These diagnostics include electrodes shielded from the beam electric field by grids, recessed capacitive electrodes, and $8 \mathrm{~cm}^{2}$ electrodes that are flush with the diagnostics tube in the fourth quadrupole. In the third quadrupole, we have flush electrodes running the length of the octagonal tube. These are divided azimuthally into two per quadrant, as shown in Fig. 4., to measure capacitive pickup from the head and tail of the beam, as well as electron emission currents between pairs of electrodes that are connected by quadrupole magnetic field lines that constrain electron flow.

Electron-clearing ring electrodes, Fig. 1, are inserted in the drift regions between quadrupole magnets, and can be biased to $+10 \mathrm{kV}$ to remove electrons from the drift regions between magnets. Another ring electrode surrounding the beam between the exit of the last quadrupole magnet and the downstream diagnostics can be biased to $-10 \mathrm{kV}$ to suppress beam-induced electrons, generated on the end structures, from reaching the magnets.

Two types of gridded electrodes are used. The griddedion collectors (GIC) are located at the minor radius where quadrupole magnetic field lines are tangent to the surface, therefore can provide magnetic suppression of electron emission from the grids or the collector; while ions, from ionization of gas, that are heavier than hydrogen are expelled across the magnetic field by the beam potential $\left(\phi_{\mathrm{b}}=2 \mathrm{kV}\right.$ in HCX). Gridded-electron collectors (GEC) are located at azimuths where quadrupole field lines enter the beam tube. Electrons that are detrapped as the beam potential falls to zero at the end of the pulse can flow along magnetic field lines from the beam to the collector. This will be discussed in more detail in the next section.

The purpose of the grids is to shield the GIC/GEC from capacitive pickup, which exceeds the expected current of expelled ions or detrapped electrons by 3 orders of magnitude. The effectiveness of the grids was tested before installation by pulsing square waves on a metal cylinder at the beam position, relative to the grounded diagnostics beam tube. The signal to gridded collectors was compared with the signal to bare collectors, scaled to the same area, to determine the shielding factor of the grids. Electromesh grids of 90 mesh/inch, $90 \%$ transparency, were sandwiched between the stainless steel diagnostics beam tube and a thin stainless steel frame, which was spot-welded around the periphery, grounding the grids to the diagnostics beam tube. A single grid provided a shielding factor of 30 , which as inadequate compared with the expected capacitive pickup. Adding a second grid, one on the inside and one on the outside of the $0.4 \mathrm{~mm}$ thick beam tube, increased the shielding factor to 400-600. In passing, we remark that good electrical connection between the grids and the beam tube is essential for effective shielding, as expected: initial tests with grids attached with conductive tape provided a shielding factor of only 10 , for either 1 or 2 grids.

Results from the GIC have been previously reported [7]. The current-voltage characteristic was flat within $10 \%$ for negative collector bias between -20 and $-60 \mathrm{~V}$. We found with the injection of argon gas that the current of expelled ions from gas increased linearly with the measured gas pressure, therefore the GIC's can be calibrated as fast ion gauges, to measure the timedependent gas pressure within the beam. The current of expelled ions is also related to the ionization rate of gas by the beam, however the current of ions from charge exchange has to be subtracted to yield the net ionization current.

\section{FIRST ELECTRON ACCUMULATION RESULTS}

The GEC's are located where magnetic field lines enter the beam tube, allowing electrons near or within the beam to flow along magnetic field lines through the grids to the collector, as the electrons are detrapped by the decreasing beam potential at the end of a pulse. The current-voltage characteristic was flat within $10 \%$ for positive collector bias between 20 and $80 \mathrm{~V}$. The effects of reversing the bias polarity are revealing: In Fig. 5, we compare the Faraday cup after the magnets, time shifted to the GEC

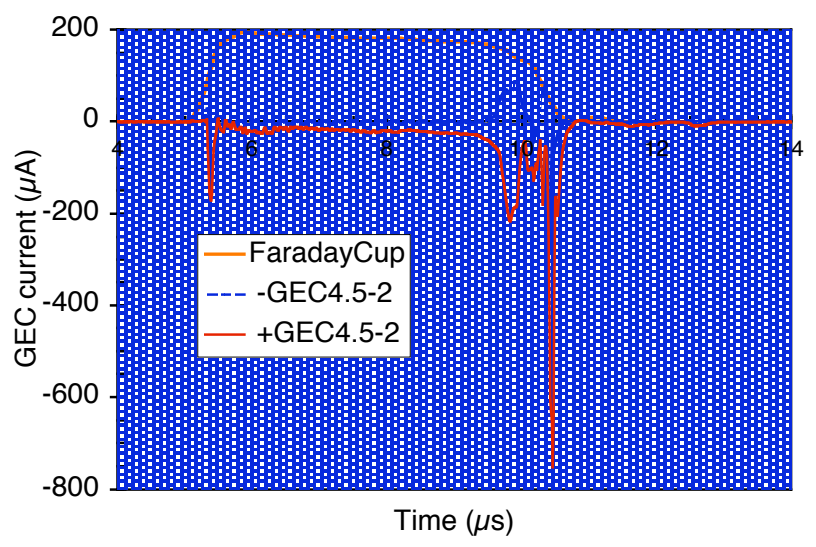

Fig. 5. The currents to a gridded electron collector (GEC) are shown for negative and positive collector bias, and are compared to the time dependence of the end Faraday cup, which is time shifted to the GEC axial position. The suppressor is biased to $\mathrm{V}_{\mathrm{s}}=-10 \mathrm{kV}$, and the three clearing electrodes are all biased to $+9 \mathrm{kV}$. 
position using capacitive pickup from the beam to the flush short collectors (FLS) at the same axial position as the GEC to calibrate the time-shift.

The negatively biased GEC current becomes positive at the head and tail of the beam - at the tail where the beam potential is falling, we attribute this to energetic beamhalo ions scattered off the opposite wall that hit the collector causing electron emission. Negative currents that occur at the same time with a positively biased GEC we attribute to electron emission from the beam tube at the opposite end of a quadrupole field line that intersects the GEC collector. These electron currents would therefore be measured as they are generated. Spikes of current from halo loss have been observed previously in the upstream electrostatic quadrupoles [8].

The spikes at the beam head, where the beam potential is rising, are less likely to come from the opposite wall, because during the electron bounce time of $8 \mathrm{~ns}$ in a 1000 $\mathrm{V}$ beam potential, the beam potential increases by $40 \mathrm{~V}$, so only electrons emitted with energies $\geq 40 \mathrm{eV}$ could reach the GEC on the opposite wall. We therefore think that the negative spike is more likely to come from halo ions hitting the grids, and generating electrons there that are attracted to the positive collector.

On the other hand, a negative current to the positively biased GEC, with no corresponding positive current at the same time to the negatively biased GEC, we attribute to electrons that have been trapped by the positive beam potential, and are released as the beam potential falls. These are deeply trapped by the beam potential, that ranges from $1 \mathrm{kV}$ at the edge of the beam to $2 \mathrm{kV}$ on axis, as predicted by beam codes [9] and measured with a retarding potential analyzer [10]. These negative currents occur during the final decay of the beam current to zero at the end of the pulse. They are seen to decay to zero at the same time as the beam current, as expected for electrons generated from ionization of gas near the beam axis.

The mid-pulse negative current to the GEC we attribute to weakly trapped electrons, emitted from the beam tube. These may be related to mid-pulse electron currents to the clearing electrodes that remain finite even with end-wall electrons suppressed. The cause of these is under active investigation.

Returning to the end-of-pulse negative current to the GEC: If these are deeply trapped electrons, probably from ionization of gas by the beam, integration of this end-ofpulse current will yield the number of trapped electrons. We can choose between positive and negative bias on the collector. Positive bias will suppress electron emission from the collector, but can collect electrons emitted from the grids, which will add to the detrapped electron current, giving an upper bound on the electron charge at the end of the pulse. If the current with negative bias is negative during this time, we conclude that beam ions are not striking the beam-tube where they would generate additional electrons. Negative bias won't suppress electron emission from the collector but will suppress electrons emitted from the grids; electron emission from the collector will subtract from the detrapped current for a lower limit on electron charge.

To obtain the electron line charge, we observe that the GEC's not only collect electrons from all magnetic flux that passes through the beam but also from most of the remaining region outside the beam. Since quadrupole fields have four similar regions azimuthally, we multiply the measured signal to one of these regions by 4. Axially, the GEC aperture is $2.8 \mathrm{~cm}$ long. We therefore can obtain the line-charge per meter from the measured electron charge from the beam tail by multiplying by $(4 / 0.028)=$ 143. With this factor, numerically integrating the negative tail spike, and dividing by the transmission of the double grid (totaling 0.77), we obtain the electron line-charges $\lambda_{\mathrm{e}}$, listed in Table 1, normalized to the ion beam charge $\left(/ \lambda_{\mathrm{b}}=0.082 \mu \mathrm{C} / \mathrm{m}\right)$. We have not yet determined the electron density with high precision, the data in Table 1 exhibit upper limits that are 4-7 times the lower limits.

Table 1. Upper and lower limits on $\lambda_{\mathrm{e}} / \lambda_{\mathrm{b}}$ (Data 11/08/04), under varying conditions of clearing electrodes $\mathrm{V}_{\mathrm{c}}$ (a-c) and suppressor electrode $\mathrm{V}_{\mathrm{s}}$ on or off, and with aperture inserted to reduce beam current from 180 to $25 \mathrm{~mA}$.

\begin{tabular}{|l|l|l|l|l|l|l|}
\hline Shot & $\mathbf{V}_{\mathbf{s}}$ & $\begin{array}{l}\mathbf{V}_{\mathbf{c}} \\
(\mathbf{a})\end{array}$ & $\begin{array}{l}\mathbf{V}_{\mathbf{c}} \\
(\mathbf{b})\end{array}$ & $\begin{array}{l}\mathbf{V}_{\mathbf{c}} \\
(\mathbf{c})\end{array}$ & $\begin{array}{l}\boldsymbol{\lambda}_{\mathbf{e}} / \boldsymbol{\lambda}_{\mathbf{b}} \\
(+\mathbf{5 0} \mathbf{V})\end{array}$ & $\begin{array}{l}\boldsymbol{\lambda}_{\mathbf{e}} / \boldsymbol{\lambda}_{\mathbf{b}} \\
(\mathbf{- 5 0} \mathbf{V})\end{array}$ \\
\hline 163625 & -10 & +9 & +9 & +9 & 0.17 & 0.037 \\
\hline 172451 & 0 & +9 & 0 & 0 & 0.31 & 0.064 \\
\hline 174030 & -10 & 0 & 0 & 0 & $\geq 0.86$ & 0.125 \\
\hline
\end{tabular}

We are continuing to compare these results against other measurements, including independent measurements of the beam potential, particularly from the energy of expelled ions from gas [10] and from integrals of electron source rates. We expect that this effort will determine the accuracy of the electron accumulation measurements and will reduce the uncertainties to acceptable levels.

\section{REFERENCES}

[1] R. H. Cohen, et al., to be published in Phys. Of Plasmas, May (2005).

[2] R. H. Cohen, "Bridging timescales for simulating electron clouds," ROPA009, PAC05.

[3] J-L. Vay, "Filling in the Roadmap for self-consistent electron cloud and gas modeling," ROPB006, PAC05.

[4] A. W. Molvik, et al., "Gas desorption and electron emission from $1 \mathrm{MeV}$ potassium ion bombardment of stainless steel," Physical Review Special Topics Accelerators and Beams 7, 093202 (2004).

[5] E. Mahner, et al., Phys. Rev. Special Topics Accelerators and Beams 6, 013201 (2003).

[6] P. Thieberger, et al., Phys. Rev. A61, 042901 (2000).

[7] A.W. Molvik, P. A. Seidl, F. M. Bieniosek, R. H. Cohen, M. Kireeff Covo, L. Prost, "Experimental Studies of Electrons in a Heavy-Ion Beam", To be 
published in Nuc. Instrum,. And Methods A. Also available as LLNL Report UCRL- JRNL-204975.

[8] L. R. Prost, P. A. Seidl, F. M. Bieniosek, C. M. Celata, A. Faltens, D. Baca, E. Henestroza, J. W. Kwan, M. Leitner, W. L. Waldron, R. Cohen, A. Friedman, D. Grote, S. M. Lund, A. W. Molvik, and E. Morse, "High current transport experiment for heavy ion inertial fusion," Physical Review Special Topics - Accelerators and Beams 8, 020101 (2005).

[9] S. Lund, private communication (2004),

[10] M. Kireeff Covo, to be published. 